\title{
Minimally invasive ileal pouch-anal anastomosis for patients with obesity: a propensity score-matched analysis
}

\author{
Mohamed A. Abd El Aziz ${ }^{1}$ (D) - Giacomo Calini ${ }^{1} \cdot$ Fabian $_{\text {Grass }}{ }^{2} \cdot$ Kevin T. Behm $^{1} \cdot{\text { Anne-Lise } D^{\prime} \text { Angelo }}^{1}$. \\ Sherief Shawki ${ }^{1}$ Kellie L. Mathis ${ }^{1}$ - David W. Larson ${ }^{1}$
}

Received: 11 March 2021 / Accepted: 10 May 2021 / Published online: 13 May 2021

(C) The Author(s), under exclusive licence to Springer-Verlag GmbH Germany, part of Springer Nature 2021

\begin{abstract}
Background Obesity is a risk factor for failure of pouch surgery completion. However, little is known about the impact of obesity on short-term outcomes after minimally invasive (MIS) ileal pouch-anal anastomosis (IPAA). This study aimed to assess shortterm postoperative outcomes in patients undergoing MIS total proctocolectomy (TPC) with IPAA in patients with and without obesity.

Materials and methods All adult patients ( $\geq 18$ years old) who underwent MIS IPAA as reported in the American College of Surgeons National Surgical Quality Improvement Program (ACS-NSQIP) Participant User Files 2007 to 2018 were included. Patients were divided according to their body mass index (BMI) into two groups $\left(\mathrm{BMI} \geq 30 \mathrm{~kg} / \mathrm{m}^{2} \mathrm{vs}\right.$. BMI $<30 \mathrm{~kg} / \mathrm{m}^{2}$ ). Baseline demographics, preoperative risk factors including comorbidities, American Society of Anesthesiologists Class, smoking, different preoperative laboratory parameters, and operation time were compared between the two groups. Propensity score matching (1:1) based on logistic regression with a caliber distance of 0.2 of the standard deviation of the logit of the propensity score was used to overcome biases due to different distributions of the covariates. Thirty-day postoperative complications including overall surgical and medical complications, surgical site infection (SSI), organ space infection, systemic sepsis, 30-day mortality, and length of stay were compared between both groups.

Results Initially, a total of 2158 patients (402 (18.6\%) obese and $1756(81.4 \%)$ nonobese patients) were identified. After 1:1 matching, 402 patients remained in each group. Patients with obesity had a higher risk of postoperative organ/space infection $(12.9 \%$; vs. $6.5 \%$; p-value 0.002$)$ compared to nonobese patients. There was no difference between the groups regarding the risk of postoperative sepsis, septic shock, need for blood transfusion, wound disruption, superficial SSI, deep SSI, respiratory, renal, major adverse cardiovascular events (myocardial infarction, stroke, cardiac arrest requiring cardiopulmonary resuscitation), venous thromboembolism, 30-day mortality, and length of stay.

Conclusion MIS IPAA can be safely performed in patients with obesity. However, patients with obesity have a 2-fold risk of organ space infection compared to patients without obesity. Loss of weight before MIS IPAA is recommended not only to allow for pouch creation but also to decrease organ space infections.
\end{abstract}

Keywords Minimally invasive surgery $\cdot$ Ileal pouch-anal anastomosis $\cdot$ Surgical site infection $\cdot$ Ulcerative colitis

This study presented in Western Surgical Association's meeting 2020 as a poster presentation.

David W. Larson

Larson.David2@mayo.edu

1 Division of Colon and Rectal Surgery, Department of Surgery, Mayo Clinic, 200 first St. Southwest, Rochester, MN 55905, USA

2 Department of Visceral Surgery, Lausanne University Hospital CHUV, University of Lausanne, Lausanne, Switzerland

\section{Introduction}

Restorative proctocolectomy with ileal pouch-anal anastomosis (IPAA) is the surgical treatment of choice for patients with ulcerative colitis (UC) who failed medical management as it improves both quality of life and functional outcomes [1, 2]. However, inadequate mesenteric length and lack of working space in the confined anatomical planes of the narrow pelvis represent technical challenges, which are directly related to increasing body mass index (BMI) [3]. Furthermore, the increasing adoption of minimally invasive surgery (MIS) 
platforms may bear challenges in this population given the loss of tactile sense related to the heavy mesentery and the fragile tissue prone to bleeding in patients with obesity [3, 4]. This might translate into worse postoperative outcomes secondary to bleeding or anastomotic leak. In this present study, we attempted to assess whether the touted benefits of MIS apply to the challenging population of obese patients undergoing IPAA surgery.

\section{Methods}

All adult patients undergoing MIS total proctocolectomy (TPC) with IPAA (Current Procedure Terminology code: 44211) for ulcerative colitis (International Classification of Diseases (ICD) codes: ICD-9-CM: 556.x and ICD-10-CM: K51.x) from 2007 to 2018 and reported in the American College of Surgeons National Surgical Quality Improvement Program (ACS-NSQIP) database were included.

The ACS-NSQIP is a validated national database for which trained data abstractors extract patients' information based on predefined data extraction sheets according to standardized definitions. Patients are identified for inclusion in the final dataset based on a random sample with approximately $20 \%$ of all cases retained [5].

Patients were divided according to their body mass index (BMI) into two groups: patients with obesity (BMI $\geq 30 \mathrm{~kg} /$ $\mathrm{m}^{2}$ ) vs. patients without obesity (BMI $\left.<30 \mathrm{~kg} / \mathrm{m}^{2}\right)$. Baseline demographics, comorbidities including diabetes mellitus, hypertension, congestive heart failure, chronic obstructive pulmonary disease, bleeding disorders, anemia, hypoalbuminemia, dyspnea, smoking, American Society of Anesthesiologists (ASA) class, chronic steroid use, recent weight loss, functional health status, and operation time were compared between both groups. Further assessed were 30-day complications according to the standardized ACS-NSQIP definitions, 30-day mortality, and length of stay.

Surgical complications included any surgical site infection (superficial defined as an infection that involves only skin or subcutaneous tissue of the surgical incision, deep defined as an infection that involves any tissue beneath skin and immediate subcutaneous fat, or organ space infection defined as an infection that involves any part of the anatomy (e.g., organ or space), other than the incision which was opened and manipulated during surgery). It also can be assigned in the case of anastomotic leakage, wound disruption, systemic sepsis (septic shock or sepsis), and/or need for blood transfusion. Medical complications included any respiratory complication (pneumonia, need for ventilation more than 2 days, unplanned intubation), any renal complication (acute renal failure or chronic renal insufficiency), vascular thromboembolism (deep venous thrombosis or pulmonary embolism), major adverse cardiovascular events (myocardial infarction, stroke, or cardiac arrest requiring cardiopulmonary resuscitation), and/or urinary tract infection.

\section{Statistical analysis}

Categorical variables were summarized as frequencies and percentages and continuous variable as median (interquartile range). A propensity score matching analysis (1:1) based on logistic regression with a caliber distance of 0.2 of the standard deviation of the logit of the propensity score using nearest neighborhood matching was used to overcome biases due to different distributions of the covariates. Covariates used in the model included age, sex, preoperative comorbidities such as diabetes mellitus, dyspnea, hypertension, congestive heart failure, chronic steroid use, more than $10 \%$ loss of weight during the last 6 months, preoperative hypoalbuminemia, and ASA class. The differences between both groups were assessed using chi-squared test or Fisher's exact test as appropriate for categorical variables and Mann-Whitney test for continuous variables. All tests were two-sided and an alpha level $<0.05$ was considered statistically significant. Statistical analysis was performed using the Statistical Package for Social Sciences (SPSS, Version 25; SPSS, Inc., Armonk, NY, USA).

\section{Results}

Initially, a total of 2158 patients, $402(18.6 \%)$ with obesity and $1756(81.4 \%)$ without obesity, were identified. After 1:1 matching, 402 patients remained in each group.

Before matching, the overall median BMI was $25.1 \mathrm{~kg} / \mathrm{m}^{2}$ (IQR 22.2-29). Patients with obesity were older and had higher rates of diabetes mellitus, dyspnea, and hypertension. Furthermore, surgical duration was significantly longer in the obese group (Table 1). After propensity score matching, baseline demographics, surgical risk factors, and operation time were balanced between the groups (Table 2). Chronic steroid use, anemia, and hypoalbuminemia were the most prevalent preoperative risk factors in both groups.

Patients with obesity had a higher rate of postoperative organ space infection (12.9\%; vs. 6.5\%; p-value 0.002) compared to patients without obesity. There was no difference between the two groups regarding overall surgical complications, postoperative sepsis, septic shock, need for blood transfusion, wound disruption, superficial surgical site infection (SSI), deep SSI, overall medical complications, respiratory, renal, major adverse cardiovascular events, venous thromboembolism, 30-day mortality, and length of stay (Table 3 ). 
Table 1 Baseline demographics, preoperative risk factors, and operation time before matching

\begin{tabular}{|c|c|c|c|}
\hline & $\mathrm{BMI}<30 \mathrm{n}=1756$ & $\mathrm{BMI} \geq 30 \mathrm{n}=402$ & P-value \\
\hline BMI, median (IQR) & $24(21.5-26.6)$ & $33(31-36)$ & $<0.0001$ \\
\hline Age; median (IQR) & $37(28-50)$ & $43(34-54)$ & $<0.0001$ \\
\hline Male & $999(56.9 \%)$ & $217(54.0 \%)$ & 0.289 \\
\hline $\mathrm{DM}$ & $48(2.7 \%)$ & $35(8.7 \%)$ & $<0.0001$ \\
\hline Current smoker & $110(6.3 \%)$ & $24(6.0 \%)$ & 0.825 \\
\hline Dyspnea & $29(1.7 \%)$ & $15(3.7 \%)$ & 0.014 \\
\hline Functional dependence & $8(0.5 \%)$ & $4(1.0 \%)$ & 0.224 \\
\hline COPD & $10(0.6 \%)$ & $4(1.0 \%)$ & 0.365 \\
\hline $\mathrm{CHF}$ & $1(0.1 \%)$ & 0 & $>0.99$ \\
\hline HTN & $157(8.9 \%)$ & $105(26.1 \%)$ & $<0.0001$ \\
\hline Chronic steroid use & $906(51.6 \%)$ & $184(45.8 \%)$ & 0.035 \\
\hline$>10 \%$ loss of weight/last 6 months & $117(6.7 \%)$ & $9(2.2 \%)$ & $<0.0001$ \\
\hline Bleeding disorder & $35(2.0 \%)$ & $11(2.7 \%)$ & 0.341 \\
\hline Preoperative blood transfusion & $12(0.7 \%)$ & $3(0.7 \%)$ & 0.892 \\
\hline $\mathrm{ASA} \geq 3$ & $420(23.9 \%)$ & $150(37.3 \%)$ & $<0.0001$ \\
\hline Operation time & $287(225-355)$ & $332(255-416)$ & $<0.0001$ \\
\hline Hypoalbuminemia & & & 0.023 \\
\hline Yes & $320(18.2 \%)$ & $51(12.7 \%)$ & \\
\hline Missing & $651(37.1 \%)$ & $157(39.1 \%)$ & \\
\hline Anemia & & & 0.723 \\
\hline Yes & $405(23.1 \%)$ & $90(22.4 \%)$ & \\
\hline Missing & $124(7.1 \%)$ & $33(8.2 \%)$ & \\
\hline Thrombocytopenia & & & 0.531 \\
\hline Yes & $27(1.5 \%)$ & $7(1.7 \%)$ & \\
\hline Missing & $145(8.3 \%)$ & $40(10.0 \%)$ & \\
\hline
\end{tabular}

Bold indicates statiscially signficant

$B M I$ body mass index, $D M$ diabetes mellitus, $H C T$ hematocrit, $C O P D$ chronic obstructive pulmonary disease, $C H F$ congestive heart failure, HTN hypertension requiring medications, ASA American Society of Anesthesiologists

\section{Discussion}

This nationwide analysis provides an insight about the impact of obesity on short-term complications after MIS TPC with IPAA for ulcerative colitis. Overall surgical and medical complications, 30-day mortality, and length of stay were comparable between patients with obesity and the matched comparative cohort of patients without obesity. However, patients with obesity had a 2-fold increased risk of organ space infection after matching for the baseline confounders. Preoperative weight loss should be encouraged to minimize organ space infections.

Up to $30 \%$ of patients with ulcerative colitis will require colectomy during their lifetime. And, about $31.5 \%$ of patients suffering from ulcerative colitis have concomitant obesity [6]. Therefore, a deep understanding of interaction of obesity with different aspects of the disease processes and its effect on treatment strategies is pivotal. Pathophysiological mechanisms related to obesity increase the prevalence of several comorbidities, which in turn either directly or indirectly impact surgical outcomes [6]. Furthermore, surgery in patients with obesity is challenging from a technical standpoint given the highly vascularized and heavy yet fragile mesentery. In patients with chronic ulcerative colitis, surgical management is further complicated by the chronic debilitating nature of the disease and the effect of chronic medical management on patients' condition and healing processes [6]. Interestingly, in our cohort, about $19 \%$ of patients who underwent MIS IPAA had a body mass index $\geq 30 \mathrm{~kg} / \mathrm{m}^{2}$, which is slightly higher than what has been reported before when both open and MIS IPAA were included [7, 8]. This might reflect the general trend to more widespread adoption of MIS in patients with obesity $[4,9,10]$. This is important in light of conflicting results in the literature about the impact of obesity on shortterm outcomes after surgery for inflammatory bowel diseases [7, 8, 11-14].

Before matching, patients with obesity had a longer operation time, which might indirectly reflect the technical 
Table 2 Baseline demographics, preoperative risk factors, and operation time after matching

\begin{tabular}{|c|c|c|c|}
\hline & $\mathrm{BMI}<30 \mathrm{n}=402$ & $\mathrm{BMI} \geq 30 \mathrm{n}=402$ & P-value \\
\hline Age; median (IQR) & $44(32-56)$ & $43(34-54)$ & 0.997 \\
\hline Male & $237(59.0 \%)$ & $217(54.0 \%)$ & 0.155 \\
\hline $\mathrm{DM}$ & $28(7.0 \%)$ & $35(8.7 \%)$ & 0.358 \\
\hline Current smoker & $34(8.5 \%)$ & $24(6.0 \%)$ & 0.172 \\
\hline Dyspnea & $13(3.2 \%)$ & $15(3.7 \%)$ & 0.848 \\
\hline Functional dependence & $5(1.2 \%)$ & $4(1.0 \%)$ & $>0.99$ \\
\hline COPD & $3(0.7 \%)$ & $4(1.0 \%)$ & $>0.99$ \\
\hline $\mathrm{CHF}$ & 0 & 0 & \\
\hline HTN & $106(26.4 \%)$ & $105(26.1 \%)$ & $>0.99$ \\
\hline Chronic steroid use & $196(48.8 \%)$ & $184(45.8 \%)$ & 0.437 \\
\hline$>10 \%$ loss of weight/last 6 months & $12(3.0 \%)$ & $9(2.2 \%)$ & 0.659 \\
\hline Bleeding disorder & $11(2.7 \%)$ & $11(2.7 \%)$ & $>0.99$ \\
\hline Preoperative blood transfusion & $3(0.7 \%)$ & $3(0.7 \%)$ & $>0.99$ \\
\hline $\mathrm{ASA} \geq 3$ & $131(32.6 \%)$ & $150(37.3 \%)$ & 0.160 \\
\hline Hypoalbuminemia & & & 0.399 \\
\hline Yes & $62(15.4 \%)$ & $51(12.7 \%)$ & \\
\hline Missing & $162(40.3 \%)$ & $157(39.1 \%)$ & \\
\hline Anemia & & & 0.534 \\
\hline Yes & $89(22.1 \%)$ & $90(22.4 \%)$ & \\
\hline Missing & $25(6.2 \%)$ & $33(8.2 \%)$ & \\
\hline Thrombocytopenia & & & 0.423 \\
\hline Yes & $6(1.5 \%)$ & $7(1.7 \%)$ & \\
\hline Missing & $30(7.5 \%)$ & $40(10.0 \%)$ & \\
\hline
\end{tabular}

$D M$ diabetes mellitus, $C O P D$ chronic obstructive pulmonary disease, $C H F$ congestive heart failure, $H T N$ hypertension requiring medications, $A S A$ American Society of Anesthesiologists class, IQR interquartile range challenges related to MIS surgery in obese patients [7, 12, 15]. In contrast to what Klos et al. reported, a non-significant difference in both operative time and estimated blood loss between patients with obesity and patients without obesity when open surgery was predominantly utilized [16]. Therefore, although MIS IPAA might have a longer operation time than open IPAA in patients with obesity, this longer operation time does not translate into higher rates of postoperative complications [17].

The high rate of organ space infection in patients with obesity cohort despite MIS is concerning and indirectly highlighting the fact that MIS does not mitigate all the risks related to IPAA surgery. The shortened mesentery associated with obesity might indirectly affect the blood supply and thus increase the leak rates despite maximized reach maneuvers and adequate length to allow successful IPAA completion. $[3,7]$. Careful preoperative evaluation of patients' condition and preoperative optimization including weight loss may be beneficial. However, preoperative conditioning also requests correction of potential nutritional deficits (also in obese patients), anemia, and steroid weaning in order to achieve a clinical benefit $[18,19]$. Indeed, obesity was found to be an independent risk factor for pouch complications [16], and comparable rates to our results regarding pelvic infection and anastomotic leak for patients with obesity undergoing IPAA have been reported before $(18.8 \%$ for patients with obesity vs. $8.1 \%$ for patients without obesity; p-value < $0.05)$ [8]. Interestingly, in their study, the higher rate of pelvic infection did not translate into worse long-term outcomes [8]. However, Fazio et al. have shown previously that pelvic sepsis or anastomotic leak were strong predictors of pouch failure [20]. Importantly, organ space infections have a detrimental effect not only on patients but also on healthcare systems by increasing length of hospital stay [21].

Unsurprisingly, patients with obesity had higher rates of different comorbidities. By balancing the baseline confounders, we tried to better understand the impact of MIS by mitigating the impact of these comorbidities. Indeed, after propensity score matching, MIS was not associated with a higher risk of medical complications, mortality, or length of stay, taking into account the differences in baseline confounders. This may highlight the benefit of MIS even in this challenging population. Indeed, the benefit of MIS in patients with obesity or even super obesity undergoing colorectal surgery has been demonstrated before [9]. 
Table 3 Postoperative complications

\begin{tabular}{llll}
\hline & BMI $<30 \mathrm{n}=402$ & BMI $\geq 30 \mathrm{n}=402$ & P-value \\
\hline Any surgical & $76(18.9 \%)$ & $91(22.6 \%)$ & 0.192 \\
SSI & $47(11.7 \%)$ & $66(16.4 \%)$ & 0.053 \\
Superficial SSI & $18(4.5 \%)$ & $11(2.7 \%)$ & 0.256 \\
Deep SSI & $5(1.2 \%)$ & $4(1.0 \%)$ & $>0.99$ \\
Organ/space SSI & $\mathbf{2 6 ( 6 . 5 \% )}$ & $\mathbf{5 2}(\mathbf{1 2 . 9 \%})$ & $\mathbf{0 . 0 0 2}$ \\
Wound disruption & $3(0.7 \%)$ & $2(0.5 \%)$ & $>0.99$ \\
Systemic sepsis & $28(7.0 \%)$ & $31(7.7 \%)$ & 0.685 \\
Sepsis & $26(6.5 \%)$ & $25(6.2 \%)$ & 0.885 \\
Septic shock & $2(0.5 \%)$ & $6(1.5 \%)$ & 0.287 \\
Need for blood transfusion & $22(5.5 \%)$ & $28(7.0 \%)$ & 0.380 \\
Any medical & $40(10.0 \%)$ & $37(9.2 \%)$ & 0.719 \\
Respiratory complications & $3(0.7 \%)$ & $7(1.7 \%)$ & 0.341 \\
Renal complications & $4(1.0 \%)$ & $4(1.0 \%)$ & $>0.99$ \\
VTE & $15(3.7 \%)$ & $17(4.2 \%)$ & 0.718 \\
MACE & $2(0.5 \%)$ & $2(0.5 \%)$ & $>0.99$ \\
UTI & $20(5.0 \%)$ & $13(3.2 \%)$ & 0.286 \\
Mortality & $1(0.2 \%)$ & $2(0.5 \%)$ & $>0.99$ \\
LOS & $6(4.75-9)$ & $6(4-9)$ & 0.906 \\
\hline
\end{tabular}

Bold indicates statiscially signficant

SSI surgical site infection, VTE venous thromboembolism, MACE major adverse cardiovascular events, UTI urinary tract infection, LOS length of stay
Our study adds to the current understanding of surgical management of patients with ulcerative colitis who have concurrent obesity. Although we could not assess long-term outcomes, surgical case volume, or experience of the surgeons, the present study represents the largest cohort in the literature reporting on the impact of obesity in MIS TPC with IPAA. Additional limitations include the retrospective nature, inability to obtain specifics on preoperative medical therapy (i.e., biologic or other immunosuppressive treatments than steroids), intraoperative complications, or whether the organ space infection is due to an anastomotic leak or other causes. In addition, no information about preoperative prehabilitation including nutritional support [18]. The impact of obesity on short-term outcomes after a two-stage operation could not be assessed due to limitations in the CPT coding. Thus, our study is confined to the group who underwent TPC from the start and the potential benefit of MIS for patients with obesity undergoing MIS three-stage IPAA could not be assessed.

\section{Conclusion}

MIS TPC with IPAA can be safely performed in patients with obesity. However, patients with obesity have a 2 -fold risk of organ space infection compared to patients without obesity. Loss of weight before MIS IPAA is recommended not only to allow for pouch creation but also to decrease organ space infections.

\section{Declarations}

Ethical approval This study utilized a national database that is Health Insurance Portability and Accountability Act (HIPAA) compliant using deidentified patients' information. Therefore, informed consent was not required.

Conflict of interest The authors declare no competing interests.

\section{References}

1. Larson DW, Cima RR, Dozois EJ, Davies M, Piotrowicz K, Barnes SA, Wolff B, Pemberton J (2006) Safety, feasibility, and short-term outcomes of laparoscopic ileal-pouch-anal anastomosis: a single institutional case-matched experience. Ann Surg 243(5):667-670 discussion 70-2

2. Larson DW, Dozois EJ, Piotrowicz K, Cima RR, Wolff BG, Young-Fadok TM (2005) Laparoscopic-assisted vs. open ileal pouch-anal anastomosis: functional outcome in a case-matched series. Dis Colon Rectum 48(10):1845-1850

3. Khasawneh MA, McKenna NP, Abdelsattar ZM, Johnson A, Dozois EJ, Pemberton JH et al (2016) Impact of BMI on ability to successfully create an IPAA. Dis Colon Rectum 59(11):1034 1038

4. Abd El Aziz MA, Grass F, Behm KT, Shawki S, D'Angelo AL, Mathis KL et al (2020) Trends of complications and innovative 
techniques' utilization for colectomies in the United States. Updat Surg

5. Shiloach M, Frencher SK Jr, Steeger JE, Rowell KS, Bartzokis K, Tomeh MG, Richards KE, Ko CY, Hall BL (2010) Toward robust information: data quality and inter-rater reliability in the American College of Surgeons National Surgical Quality Improvement Program. J Am Coll Surg 210(1):6-16

6. Seminerio JL, Koutroubakis IE, Ramos-Rivers C, Hashash JG, Dudekula A, Regueiro M, Baidoo L, Barrie A, Swoger J, Schwartz M, Weyant K, Dunn MA, Binion DG (2015) Impact of obesity on the management and clinical course of patients with inflammatory bowel disease. Inflamm Bowel Dis 21(12):28572863

7. McKenna NP, Mathis KL, Khasawneh MA, Dozois EJ, Larson DW, Pemberton JH et al (2017) Obese patients undergoing ileal pouch-anal anastomosis: short-and long-term surgical outcomes. Inflamm Bowel Dis 23(12):2142-2146

8. Kiran RP, Remzi FH, Fazio VW, Lavery IC, Church JM, Strong SA, Hull TL (2008) Complications and functional results after ileoanal pouch formation in obese patients. J Gastrointest Surg 12(4):668-674

9. Abd El Aziz MA, Grass F, Perry W, Behm KT, Shawki SF, Larson DW et al (2020) Colectomy for patients with super obesity: current practice and surgical morbidity in the United States. Surg obes Relat Diseases

10. Abd El Aziz MA, Grass F, Behm KT, Dangelo AL, Mathis KL, Dozois EJ et al (2021) Trends and consequences of surgical conversion in the United States. Surg Endosc

11. Klos CL, Safar B, Jamal N, Hunt SR, Wise PE, Birnbaum EH, Fleshman JW, Mutch MG, Dharmarajan S (2014) Obesity increases risk for pouch-related complications following restorative proctocolectomy with ileal pouch-anal anastomosis (IPAA). J Gastrointest Surg 18(3):573-579

12. Canedo JA, Pinto RA, McLemore EC, Rosen L, Wexner SD (2010) Restorative proctectomy with ileal pouch-anal anastomosis in obese patients. Dis Colon Rectum 53(7):1030-1034

13. Guardado J, Carchman E, Danicic AE, Salgado J, Watson AR, Celebrezze JP, Medich DS, Holder-Murray J (2016) Obesity does not impact perioperative or postoperative outcomes in patients with inflammatory bowel disease. J Gastrointest Surg 20(4):725-733

14. Larson DW, Abd El Aziz MA, Perry W, Behm KT, Shawki S, Mandrekar J et al (2021) Surgical resection for Crohn's and cancer: a comparison of disease-specific risk factors and outcomes. Dig Surg: $1-8$

15. Efron JE, Uriburu JP, Wexner SD, Pikarsky A, Hamel C, Weiss EG, Nogueras JJ (2001) Restorative proctocolectomy with ileal pouch anal anastomosis in obese patients. Obes Surg 11(3):246251

16. Klos CL, Safar B, Jamal N, Hunt SR, Wise PE, Birnbaum EH, Fleshman JW, Mutch MG, Dharmarajan S (2013) Obesity increases risk for pouch-related complications following restorative proctocolectomy with ileal pouch-anal anastomosis (IPAA). J Gastrointest Surg 18(3):573-579

17. Mineccia M, Cravero F, Massucco P, Portigliotti L, Bertolino F, Daperno M, Ferrero A (2018) Laparoscopic vs open restorative proctocolectomy with IPAA for ulcerative colitis: impact of surgical technique on creating a well functioning pouch. Int J Surg 55: 201-206

18. Larson DW, Abd El Aziz MA, Perry W, D'Angelo AL, Behm KT, Mathis KL et al (2020) Additional value of preoperative albumin for surgical risk stratification among colorectal cancer patients. Ann Nutr Metab 76(6):422-430

19. Grass F, Pache B, Martin D, Hahnloser D, Demartines N, Hübner M (2017) Preoperative nutritional conditioning of Crohn's patientssystematic review of current evidence and practice. Nutrients 9(6)

20. Fazio VW, Tekkis PP, Remzi F, Lavery IC, Manilich E, Connor J, Preen M, Delaney CP (2003) Quantification of risk for pouch failure after ileal pouch anal anastomosis surgery. Ann Surg 238(4): 605-614 discussion 14-7

21. Martin D, Hübner M, Moulin E, Pache B, Clerc D, Hahnloser D, Demartines N, Grass F (2018) Timing, diagnosis, and treatment of surgical site infections after colonic surgery: prospective surveillance of 1263 patients. J Hosp Infect 100(4):393-399

Publisher's note Springer Nature remains neutral with regard to jurisdictional claims in published maps and institutional affiliations. 